\title{
Spatial distribution of malaria in Peninsular Malaysia from 2000 to 2009
}

\author{
Haridah Alias ${ }^{*}$, Johari Surin ${ }^{1}$, Rohela Mahmud', Aziz Shafie², Junaidden Mohd Zin³ ${ }^{3}$ Mahadzir Mohamad Nor ${ }^{3}$, \\ Ahmad Shah Ibrahim ${ }^{3}$ and Christina Rundi ${ }^{3}$
}

\begin{abstract}
Background: Malaria is still an endemic disease of public health importance in Malaysia. Populations at risk of contracting malaria includes indigenous people, traditional villagers, mobile ethnic groups and land scheme settlers, immigrants from malaria endemic countries as well as jungle workers and loggers. The predominant species are Plasmodium falciparum and $P$. vivax. An increasing number of $P$. knowlesi infections have also been encountered. The principal vectors in Peninsular Malaysia are Anopheles maculatus and An. cracens. This study aims to determine the changes in spatial distribution of malaria in Peninsular Malaysia from year 2000-2009.
\end{abstract}

Methods: Data for the study was collected from Ministry of Health, Malaysia and was analysed using Geographic Information System (GIS).

Results: Changes for a period of 10 years of malaria spatial distribution in 12 states of Peninsular Malaysia were documented and discussed. This is illustrated by digital mapping according to five variables; incidence rate (IR), fatality rate (FR), annual blood examination rate (ABER), annual parasite index (API) and slide positivity rate (SPR).

Conclusion: There is a profound change in the spatial distribution of malaria within a 10-year period. This is evident from the digital mapping of the infection in Peninsular Malaysia.

\section{Background}

Worldwide, there are 109 malaria endemic countries with an estimated 3.3 billion people at risk of malaria in 2010, out of which 1.2 billion are at high risk ( $>1$ case/ 1000 population). Of the 1.2 billion, $37 \%$ reside in Southeast Asia [1]. Malaysia was highly endemic with malaria, and in 1892, nearly one third of all deaths in Penang (an island state on the north-west of Malaysia) were attributed to malaria. Introduction of a new drainage system scheme by one of the pioneer antimalaria workers, i.e. Malcolm Watson in Peninsular Malaysia in the early twentieth century, was recognised as an early control measure in the world, leading to great reduction of malaria cases [2].

Being one of the major parasitic diseases in Malaysia, malaria affects indigenous people, traditional villagers, mobile ethnic groups and land scheme settlers, immigrants from malaria endemic countries as well as jungle

\footnotetext{
* Correspondence: firerbreathing@gmail.com

'Department of Parasitology, Faculty of Medicine, University of Malaya, 50603 Kuala Lumpur, Malaysia

Full list of author information is available at the end of the article
}

workers and loggers $[3,4]$. The total number of malaria cases in Peninsular Malaysia is taking the downward trend from 3918 in 2000 to 757 in 2005. Since then, increasing trends were observed from 2006 with 852 cases, 2007 with 1106 cases and 2008 with 1342 cases. A total of 1175 cases were reported in 2009 (Figure 1) [Unpublished data from Vector Borne Disease Division Ministry of Health records]. Malaria was rarely reported in urban areas and Kuala Lumpur only reported 15 to 20 malaria cases from 2000 to 2007. However, in 2008 and 2009, an increased figure was observed with 27 and 49 cases respectively ([3], Unpublished data from Disease Control Division, Ministry of Health records). Recently, due to rapid influx of legal and illegal immigrant workers as well as a large number of tourist into this country, they became a new source of infection which resulted in the increase in detection of malaria cases in the Klang Valley area [5]. The total number of malaria cases among immigrants was 519 in 2006 and this increased to 690 in 2009 with 49.6\% Indonesians, 17.1\% Myanmarese, 11.2\% Bangladeshis and $8.1 \%$ Thais. The remaining $14 \%$ of malaria cases were reported among immigrants from 


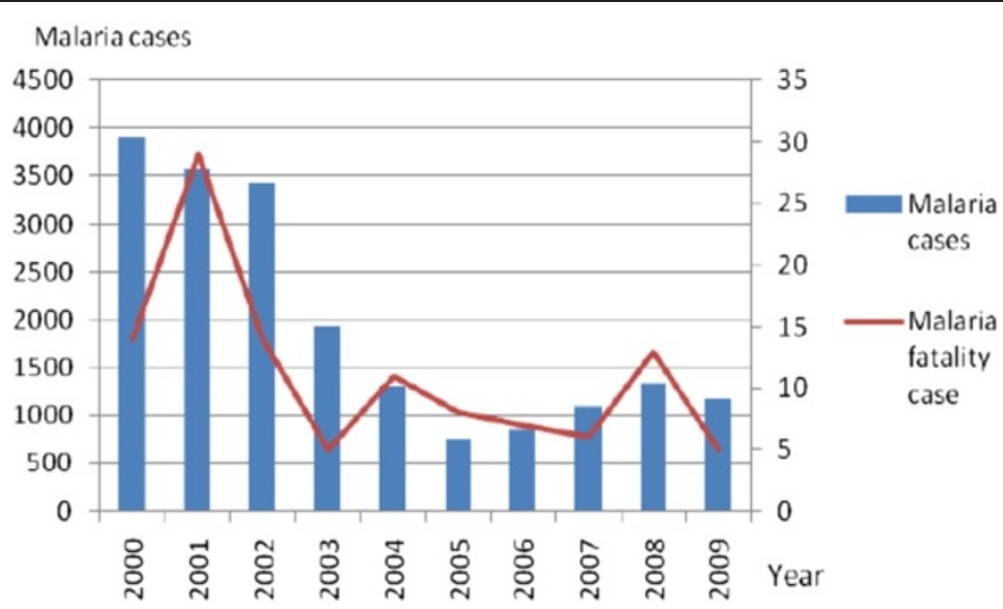

Figure 1 Malaria cases and fatality cases in Peninsular Malaysia from 2000 to 2009. Figure 1 shows the malaria cases and fatality cases reported in Peninsular Malaysia within ten years (2000 to 2009).

Pakistan, India, Nepal, Vietnam and Kampuchea (Unpublished data from Vector Borne Disease Division Ministry of Health records).

The highest number of malaria deaths in Peninsular Malaysia was reported to be 29 in 2001 and this figure decreased to 5 in 2009. The highest malaria incidence was recorded in the 20-39 years old age group ([3], Unpublished data from Disease Control Division Ministry of Health records). The younger age group who are actively working and highly mobile has increased the risk of being exposed to malaria infection, subsequently affecting the economic productivity due to work absenteeism. There were more males infected with malaria compared to females probably due to the former being involved in outdoor activities ([3,6], Unpublished data from Disease Control Division Ministry of Health records).

Five Plasmodia species are known to infect humans, namely Plasmodium falciparum, $P$. vivax, $P$. ovale, P. malariae and P. knowlesi (simian malaria parasite). In Peninsular Malaysia, in year 2009, P. vivax is the predominant species detected with (49.5\%), P. falciparum (36.1\%), P. malariae (7.2\%) and mixed infection (2.6\%) (Figure 2). P. knowlesi had shown an increasing trend with 41 cases in 2008 to 55 cases in 2009 (Unpublished data from Disease Control Division, Ministry of Health records). Chloroquine and Fansidar resistance to P. falciparum has been reported in Peninsular Malaysia [7].

Important vectors involved in malaria transmission in Peninsular Malaysia are Anopheles maculatus and An.
- P.falciparum

P.vivax

P.malariae

P.knowlesi

P.ovale

Mixed Infection

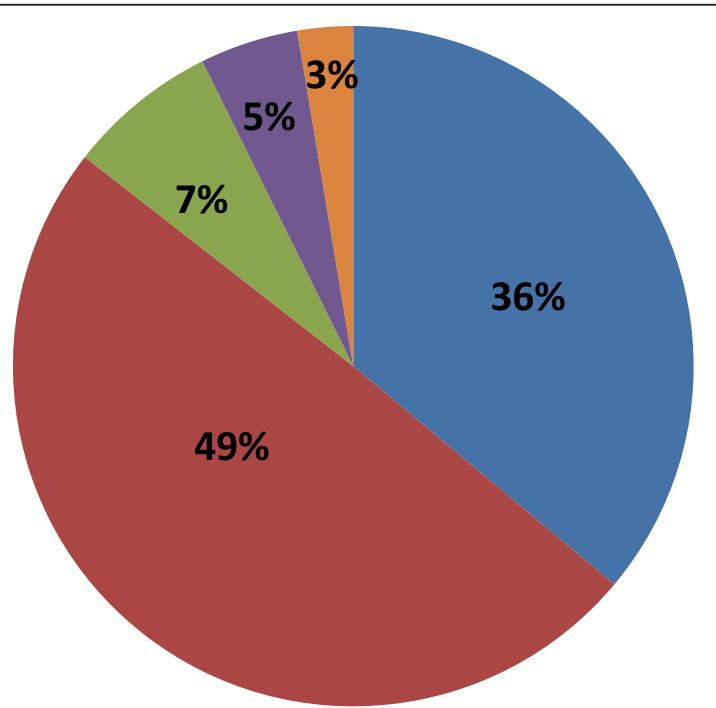

Figure 2 Percentage of malaria cases by parasite species in 2009. The percentage of malaria cases by five parasite species; Plasmodium falciparum, P. vivax, P. malariae, P. ovale, P. knowlesi and mixed infections. 
cracens. An. maculatus is the vector for human malaria whereas $A n$. cracens is the vector for simian malaria caused by P. knowlesi [8]. With deforestation and environmental changes, new vectors have displaced the established vectors.

\section{Methods}

\section{Study site}

Peninsular Malaysia stretches between $1^{\circ} 20^{\prime} \mathrm{N}$ to $6^{\circ} 40^{\prime} \mathrm{N}$ and from longitude $99^{\circ} 35^{\prime} \mathrm{E}$ to $104^{\circ} 20^{\prime} \mathrm{E}$. It covers $131,598 \mathrm{~km}^{2}$ of geographical area. Peninsular Malaysia comprised 23.5 million people as per provisional data of 2012 census. Mean daily temperatures range from about $25^{\circ} \mathrm{C}$ to $28^{\circ} \mathrm{C}$. The climate is equatorial, with rain from both the northeast (November to March) and southwest (May to August) monsoons. The rainfall ranges from a maximum of $5000 \mathrm{~mm}$ and a minimum of $1750 \mathrm{~mm}$. The average maximum relative humidity of the air varies between $94 \%$ and $100 \%$, typical of the humid tropics.
Peninsular Malaysia consists of 12 states; Perlis, Kedah, Pulau Pinang, Perak, Melaka, Negeri Sembilan, Selangor, Johor, Pahang, Terengganu, Kelantan and Federal Territory (Kuala Lumpur and Putrajaya) (Figure 3) [9].

\section{Study design}

The study was conducted using retrospective secondary data of malaria for the period of 2000 to 2009. We obtained all annual reports of malaria for the mentioned period from the Ministry of Health $(\mathrm{MOH})$ Malaysia. Besides that, the data was also obtained from public health centers and local journals. For the updated population numbers, the data was taken from Department of Statistics (DOS) Malaysia which is available online. The approval from Malaysia National Health Institute $(\mathrm{NIH})$ was successfully obtained in order to conduct the research in $\mathrm{MOH}$. A second approval was given by $\mathrm{MOH}$ to allow the data collection process. Collected data was then entered in Microsoft Excel computer program.

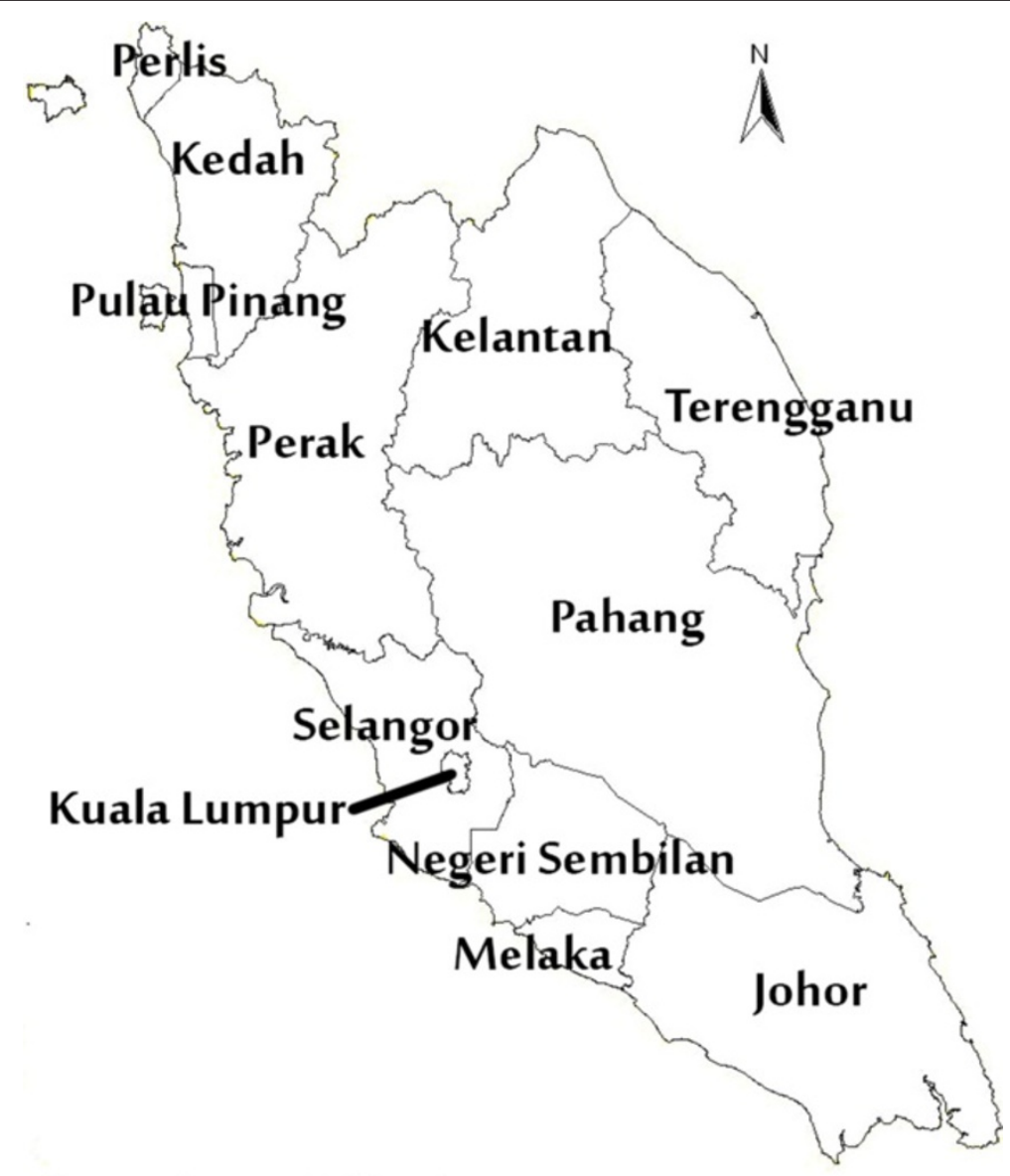

$70 \quad 0 \quad 70$ Kilometers

Figure 3 Peninsular Malaysia map. Figure 3 shows the 12 states in Peninsular Malaysia. Perlis, Kedah, Pulau Pinang and Perak situated at the northern part, Melaka, Negeri Sembilan, Federal Territory (Kuala Lumpur and Putrajaya) and Selangor at the central part, Pahang, Terengganu and Kelantan at east coast part and Johor at the southern part. 
After proper data cleaning, the data was analyzed using Geographic Information System (GIS) to produce digital maps of incidence rate (IR), fatality rate (FR), annual blood examination rate (ABER), annual parasite index (API) and slide positivity rate (SPR).

\section{Results and discussion}

Changes for a period of ten years of malaria spatial distribution in 12 states of Peninsular Malaysia are illustrated by digital mapping according to five parameters; IR, FR, ABER, API and SPR.

The changes of malaria IR for 12 states in Peninsular Malaysia from 2000 to 2009 is graphically shown in Figure 4. Within 10 years duration, all states except Pahang reported malaria IR $<10 / 10000$ population. Pahang reported malaria IR $>10 / 10000$ population from 2000 until 2003. By 2000, Perlis, Kedah, Kuala Lumpur, Selangor and Melaka had virtually eliminated malaria ([3], Unpublished data from Disease Control Division, Ministry of Health records). The areas are stratifically colour coded based on the following criteria: (i) Red (malarious area): $>10 /$ 10000 population, (ii) Yellow (malaria prone): $<10 /$ 10000 population and (iii) Green (malaria free): no local transmission [10]. Distribution of insecticide treated nets (ITNs) and indoor residual spraying (IRS) to vulnerable areas successfully decreased the malaria cases in Peninsular Malaysia [3].

Figure 5 shows the changes in FR for 12 states in Peninsular Malaysia from 2000 to 2009. There was no malaria fatality cases reported in Perlis. In Melaka, out of 15 confirmed malaria cases in 2001, two deaths were reported (13.33\%). Kuala Lumpur only reported one fatality case (6.67\%) from its total of 15 confirmed malaria cases in 2004. While in 2005, Negeri Sembilan reported one fatality case $(9.09 \%)$ from its total of 11 confirmed malaria cases. In Kedah, Pulau Pinang, Perak, Pahang, Selangor, Kelantan, Johor and Terengganu the FR was $<5 \%$ population within a 10 year period except for 2006 when Perak reported FR of 5.88\% ([3], Unpublished data from Disease Control Division Ministry of Health records). Most of the deaths were caused by cerebral and complicated malaria. Factors that contributed to malaria deaths included delay in detecting malaria due to lack of suspicion of malaria when patient was first seen, failure in detecting severe and complicated malaria which led to improper treatment, delay in treating patient with IV Quinine because of no storage in District Hospital and cases were sometimes misdiagnosed as dengue, septicemia, typhoid and hepatitis [3].

Figure 6 shows the changes of ABER in 12 states of Peninsular Malaysia from 2000 to 2009. ABER reflects the efficiency and adequacy of case detection mechanisms. There are three mechanisms of malaria case detection in Peninsular Malaysia; passive case detection (PCD), active case detection (ACD) and mass blood survey (MBS) [3]. Within a 10 year period, the values of ABER in Pahang were constantly reported as more than $10 \%$. Meanwhile, Negeri Sembilan reported ABER of $>10 \%$, twice $(2000$ and 2001). Perlis, Kedah, Pulau Pinang, Melaka, Selangor, Perak, Kelantan, Johor and Terengganu reported ABER

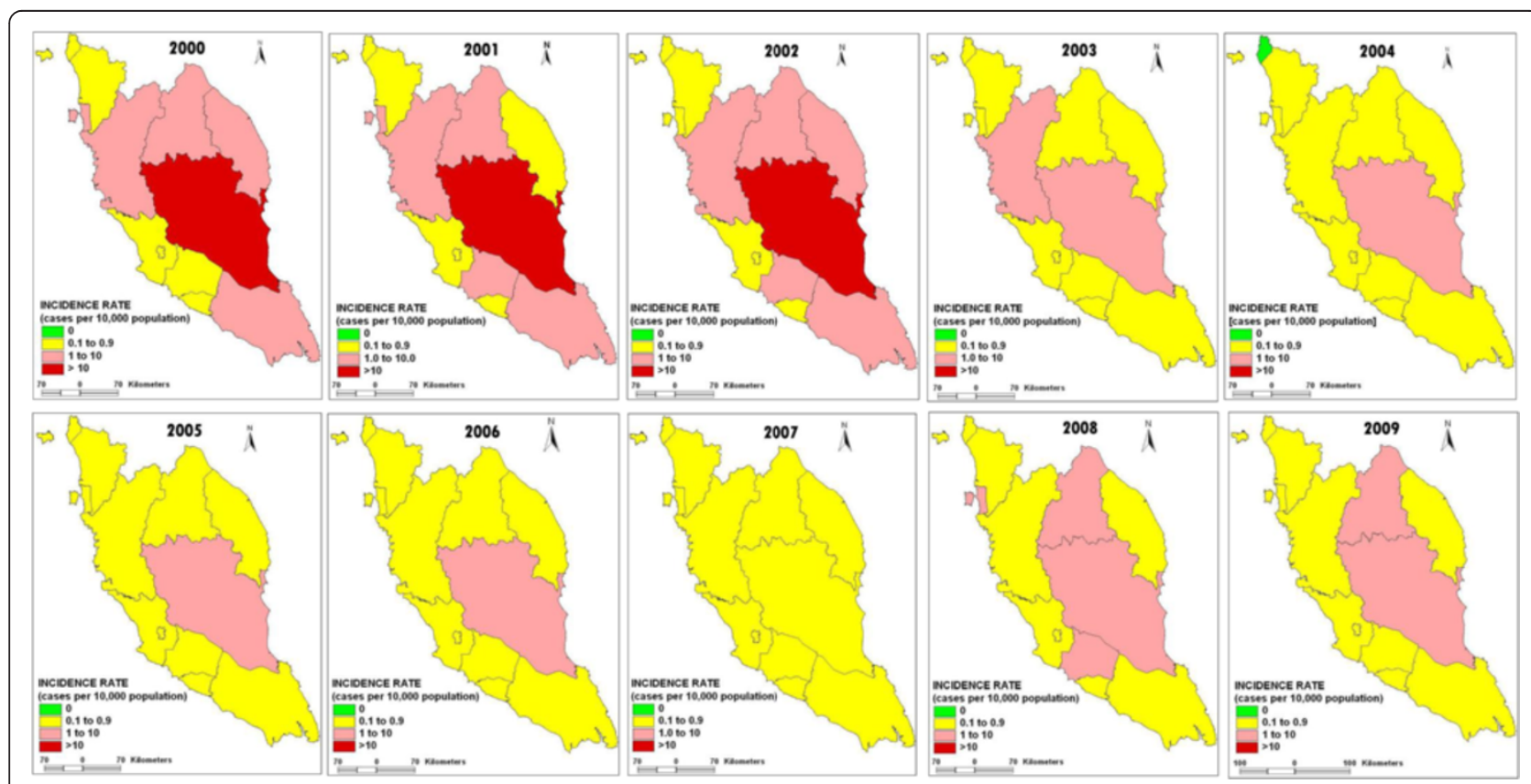

Figure 4 The changes of malaria incidence rate (IR) in 12 states of Peninsular Malaysia from 2000 to 2009. Figure 4 shows the changes of IR in 12 states of Peninsular Malaysia from 2000 to 2009. 


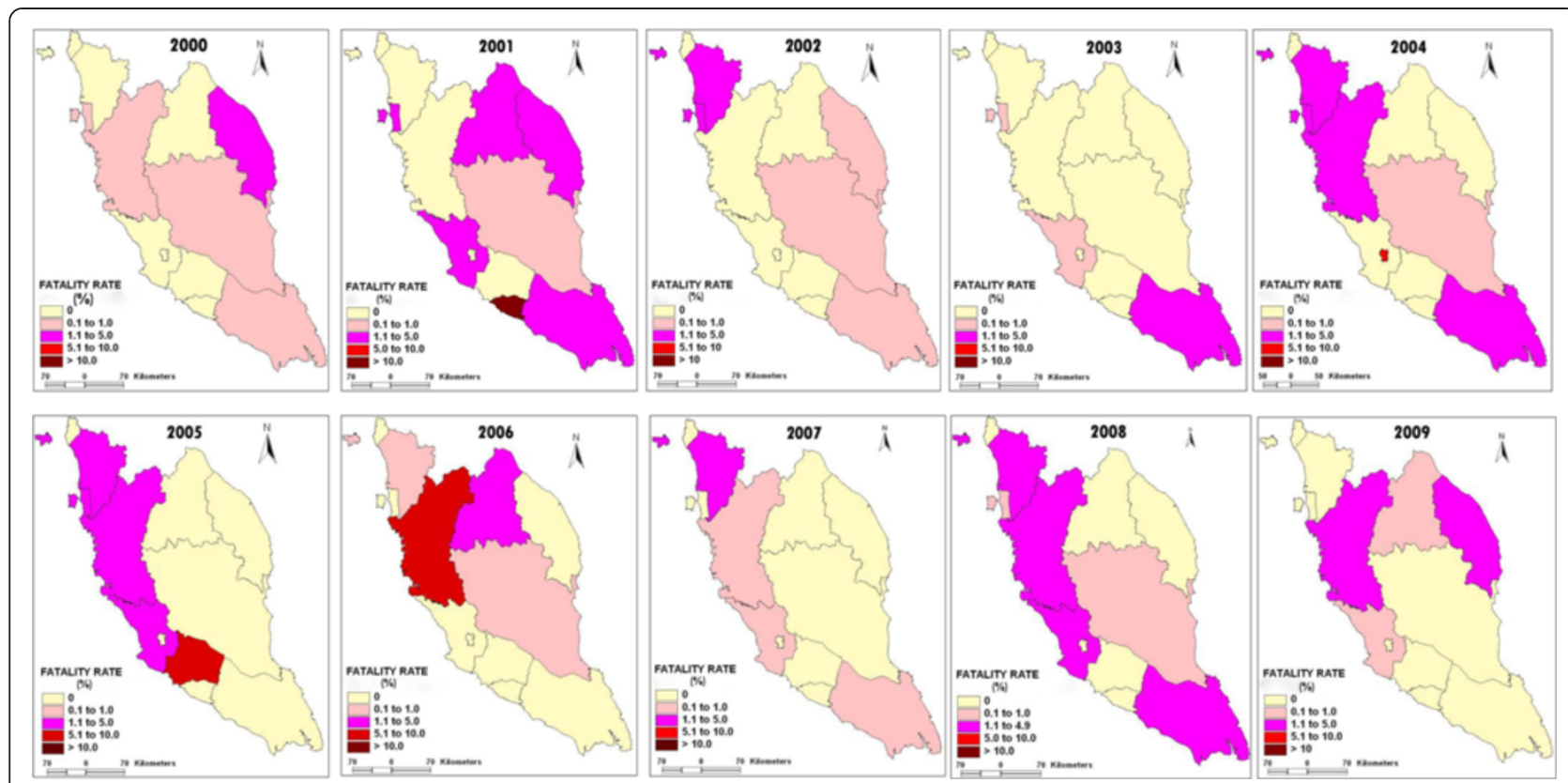

Figure 5 The changes of malaria fatality rate (FR) in 12 states of Peninsular Malaysia from 2000 to 2009. Figure 5 shows the changes of FR in 12 states of Peninsular Malaysia from 2000 to 2009.

values of less than $10 \%$ for a 10 year period. Kuala Lumpur reported the lowest ABER values of zero \% from 2000 to 2008 that increased slightly to $0.1 \%$ in 2009 ([3], Unpublished data from Vector Borne Disease Division Ministry of Health records). The value of ABER was fixed at more than $10 \%$ per year under The National Malaria Elimination Program (NMEP) (Unpublished data from Disease Control Division, Ministry of Health records). By looking at the values, most of the states in Peninsular Malaysia still have an inadequate and inefficient case detection mechanism.

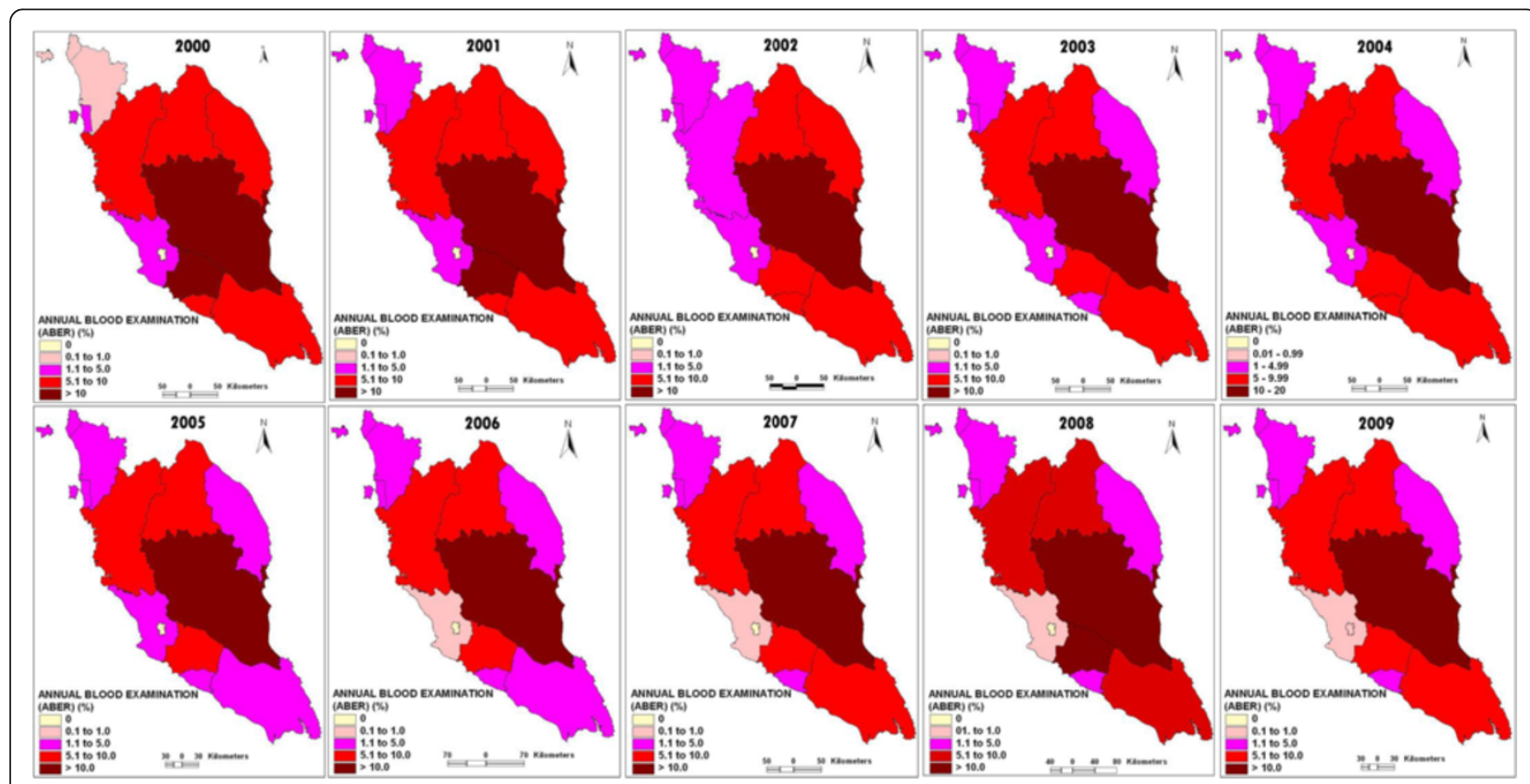

Figure 6 The changes of Annual Blood Examination Rate (ABER) in 12 states of Peninsular Malaysia from 2000 to 2009. Figure 6 shows the changes of ABER in 12 states of Peninsular Malaysia from 2000 to 2009. 


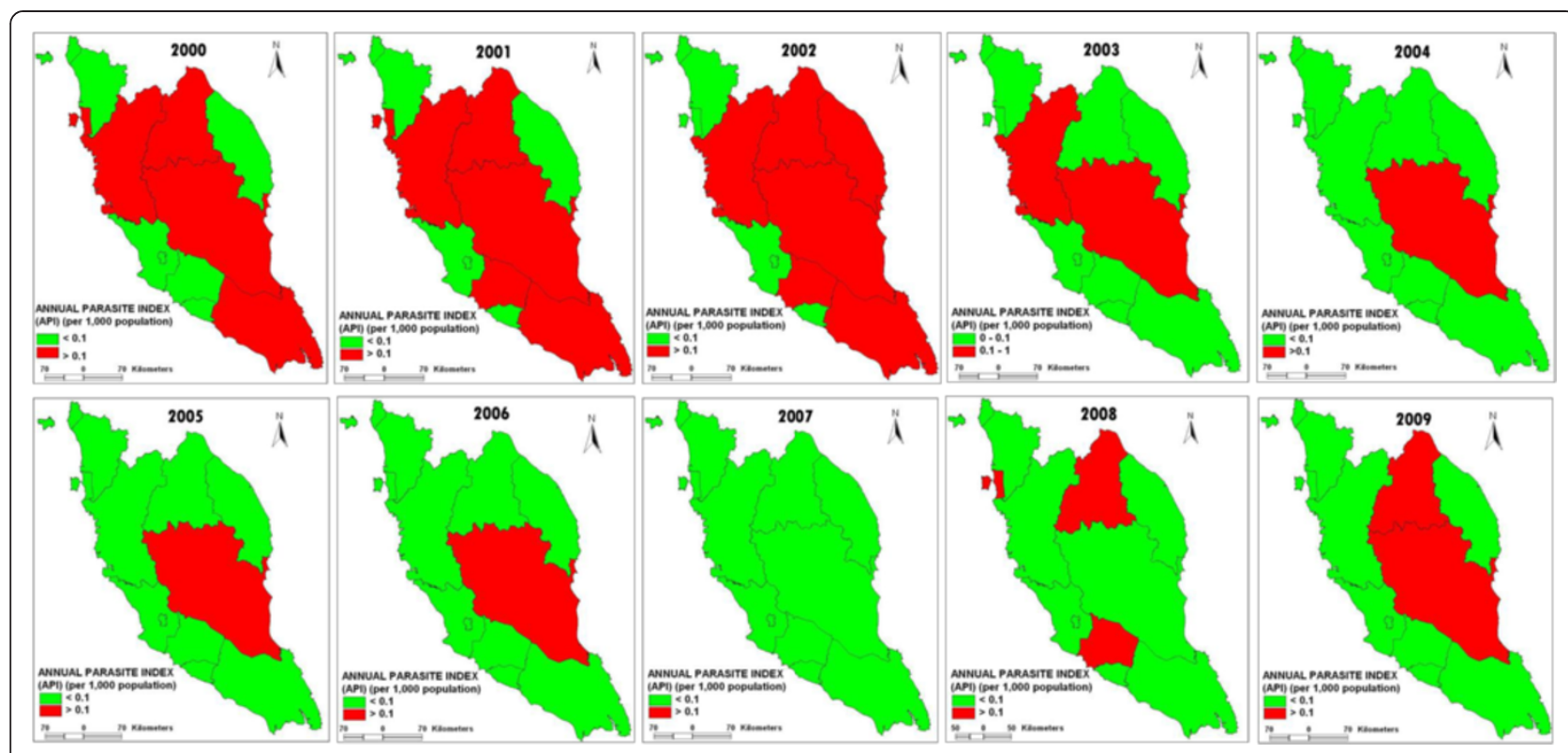

Figure 7 The changes of Annual Parasitic Index (API) in 12 states of Peninsular Malaysia from 2000 to 2009. Figure 7 shows the changes of API in 12 states of Peninsular Malaysia from 2000 to 2009.

The changes in API in 12 states in Peninsular Malaysia from 2000 to 2009 are shown in Figure 7. API depends on the adequacy of case detection mechanism i.e. ABER. If $A B E R$ is adequate, this parameter is the most important criterion to assess the progress of the eradication program. Perlis, Kedah, Selangor, Kuala Lumpur and Melaka reported API values of $<0.1 / 1000$ population for a 10 year period. Perak and Johor reported a decline in API values of $>0.1 / 1000$ population to $<0.1 / 1000$ population after the first four years. From 2000-2009, Pahang reported API values of $>0.1 / 1000$ population, except in 2008 , the value is $<0.1 / 1000$ population. Pulau Pinang, Negeri Sembilan, Terengganu and Kelantan reported varied API values ranging from $<0.1$ and $>0.1 / 1000$

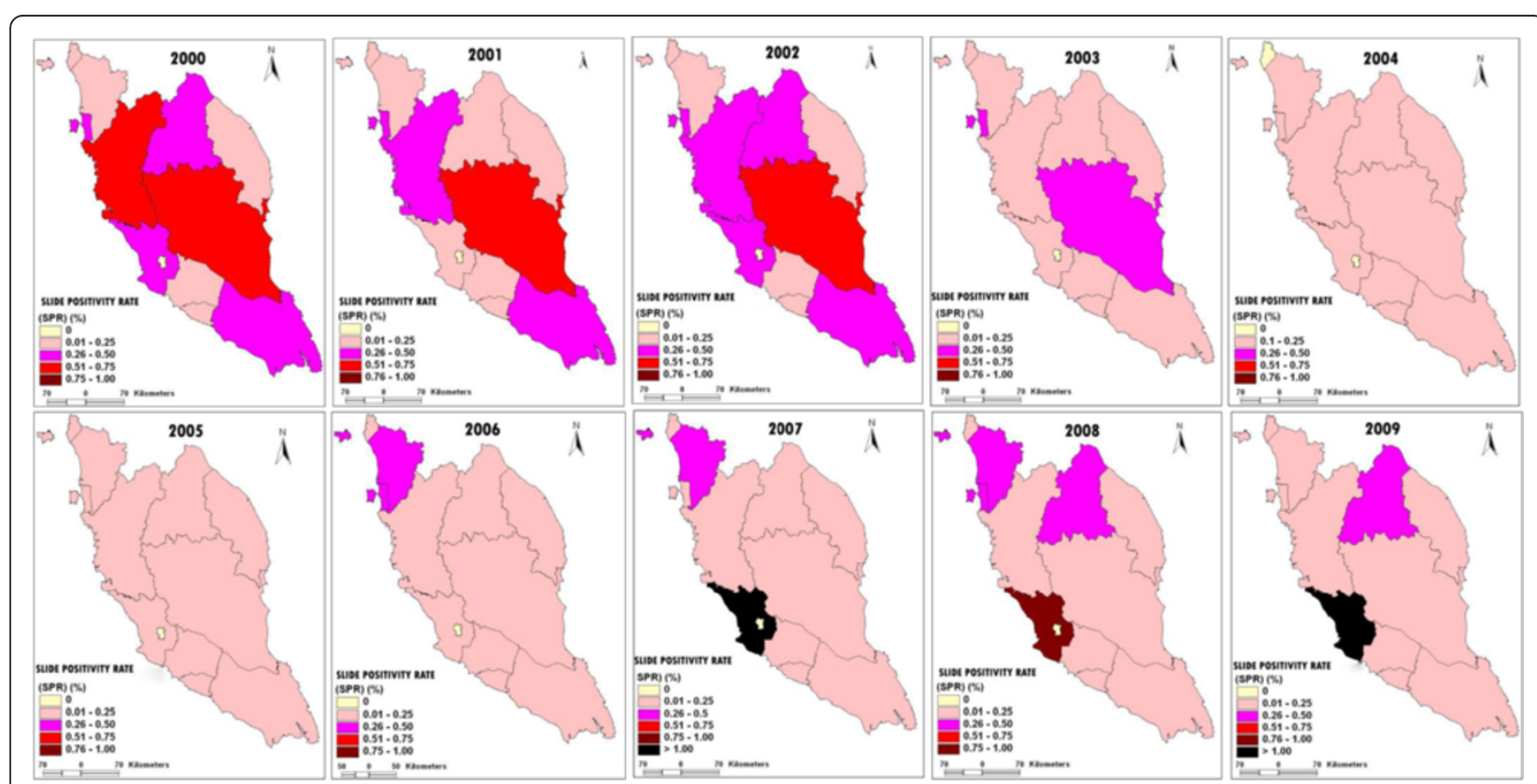

Figure 8 The changes of Slide Positivity Rate (SPR) in 12 states of Peninsular Malaysia from 2000 to 2009. Figure 8 shows the changes of SPR in 12 states of Peninsular Malaysia from 2000 to 2009. 
population ([3], Unpublished data from Disease Control Division, Ministry of Health records). Under NMEP, API value was fixed at $<0.1 / 1000$ population. To achieve the target, the area with high API values must be given priority and attention in terms of malaria control activities [11].

Figure 8 shows the changes in SPR in 12 states in Peninsular Malaysia from 2000 to 2009. Whenever ABER is adequate, SPR is a dependable parameter for determining the progress of measures and gives information of parasitic load in the community. SPR measures the prevalence of malaria parasites among those who seek care and are examined in health facilities [11]. From 2000 to 2009, all states reported SPR values $<1 \%$ except for Selangor and Kuala Lumpur. The SPR values were reported $>1 \%$ (1.1\%) in 2007 (Selangor) and 2009 (Selangor (1.04\%) and Kuala Lumpur (4.98\%) ([3], Unpublished data from Disease Control Division Ministry of Health records). In 2007, Selangor reported the highest malaria cases with 330 cases, mostly attributable to imported cases in immigrant workers. Within a10 year period (2000-2009), Kuala Lumpur recorded the highest malaria cases (49) in 2009, out of which 39 were imported cases [4]. WHO guidelines consider a country is ready to undergo transition from control to pre-elimination when the SPR value $<5 \%$ [11]. Malaysia is entering a pre-elimination stage and Peninsular Malaysia is targeted for malaria elimination by 2015 [4]. To achieve elimination, several strategies were implemented such as early case detection and prompt treatment, close monitoring of imported cases, residual spraying, usage of bed nets, environmental and anti larval management, monitoring of drug resistance and collaborating with the neighbouring countries [10].

\section{Conclusion}

There is a profound change in the spatial distribution of malaria within a 10 -year period. This is evident from the digital mapping of the infection in Peninsular Malaysia. The reduction in the number of malaria indigenous cases has been attributed to a successful Malaria Control Program (1980), which includes the increase in laboratory diagnostic capability, prompt treatment, nationwide implementation of insecticide treated bed nets and regular insecticide spraying.

\section{Competing interests}

The authors declare that they do not have competing interests.

\section{Authors' contributions}

HA conceived the study and wrote the manuscript. HA, MMN and ASI performed the data collection. AS developed the GIS map. JS, RM, AS, JMC and $C R$ revised the manuscript and gave approval of the version to be published. All authors read and approved the final manuscript.

\section{Acknowledgements}

The authors would like to thank the Director of Health Malaysia for permission to publish this paper. We gratefully acknowledge The Ministry of
Health Malaysia for supplying the data as well as the $\mathrm{MOH}$ staff who helped to collect the data. The research is funded by HIR grant (E000051-20001), Ministry of Higher Education Malaysia and UMRG grant (222110HTM).

\section{Author details}

'Department of Parasitology, Faculty of Medicine, University of Malaya, 50603 Kuala Lumpur, Malaysia. ${ }^{2}$ Department of Geography, Faculty of Arts \& Social Sciences, University of Malaya, 50603 Kuala Lumpur, Malaysia. ${ }^{3}$ Ministry of

Health Malaysia, Kuala Lumpur, Malaysia.

Received: 16 January 2014 Accepted: 10 April 2014

Published: 15 April 2014

\section{References}

1. World Health Organization: World Malaria Report 2011. Geneva: World Health Organization; 2011.

2. Sandosham AA: Malariology with special reference to Malaya. Malaysia: University of Malaya Press; 1965.

3. Ministry of Health Malaysia: Annual Report 2000-2005. Disease Control Division. Malaysia: Ministry of Health; 2005.

4. MDG 6: Combat HIV, malaria and other diseases. Malaysia: The new development goals at 2010. Kuala Lumpur: UNCT/EPU (United Nations Country Team and Economic Planning Unit); 2010.

5. Jamaiah I, Khairul Anuar A, Najib, Zurainee MN: Imported Malaria: A Retrospective study in University Hospital, Kuala Lumpur, A ten year experience. Med J Malay 1999, 53:6-9.

6. Sidhu PS, Ng SC: A Retrospective Study on Malaria Cases Admitted to the University Hospital, Kuala Lumpur, 1984-1988. Med J Malay 1991, 46:177-182.

7. World Health Organization: Review of the malaria drug efficacy situation in 10 countries of the WHO Western Pacific Region, 1987 - 2003. Geneva: World Health Organization; 2005.

8. Vythilingam I: Plasmodium knowlesi and Wuchereria bancrofti : their vectors and challenges for the future. Front Physiol 2012, 3:115.

9. ICID- Irrigation and Drainage in the World: A Global Review. 2012. http://www.icid.org/i_d_malaysia.pdf.

10. World Health Organization: World Malaria Report 2008. Geneva: World Health Organization; 2008.

11. Feachem RGA and the Malaria Elimination Group: Shrinking the Malaria Map. A Guide on Malaria Elimination for Policy Makers. San Francisco: The Global Health Group, Global Health Sciences, University of California, San Francisco; 2009.

doi:10.1186/1756-3305-7-186

Cite this article as: Alias et al: Spatial distribution of malaria in

Peninsular Malaysia from 2000 to 2009. Parasites \& Vectors 2014 7:186.

\section{Submit your next manuscript to BioMed Central and take full advantage of:}

- Convenient online submission

- Thorough peer review

- No space constraints or color figure charges

- Immediate publication on acceptance

- Inclusion in PubMed, CAS, Scopus and Google Scholar

- Research which is freely available for redistribution 